Research Paper

\title{
Systemic immune-inflammation index (SII): A More Promising Inflammation-Based Prognostic Marker for Patients with synchronic colorectal peritoneal
}

\section{carcinomatosis}

\author{
Qian Yan ${ }^{1,2^{*}}$, Zhai Ertao ${ }^{1,2^{*}}$, Zhang Zhimei ${ }^{3}$, Dai Weigang ${ }^{1,2}$, Peng Jianjun ${ }^{1,2}$, Chen Jianhui ${ }^{1,2}{ }^{\bowtie}$, Chen \\ Chuangqi ${ }^{2}$ \\ 1. Division of Gastrointestinal Surgery Center, The First Affiliated Hospital, Sun Yat-sen University, Guangzhou 510080, China. \\ 2. Gastric Cancer Center, Sun Yat-sen University, Guangzhou 510080, China. \\ 3. Department of Pathology, The First Affiliated Hospital, Sun Yat-sen University, Guangzhou 510080, China. \\ *Qian Yan and Zhai Ertao contributed equally to this work. \\ $\square$ Corresponding author: Dr. Chen Jianhui and Chen Chuangqi, Division of Gastrointestinal Surgery Center, The First Affiliated Hospital of Sun Yat-sen \\ University, Guangzhou 510080, China, Phone \& Fax number: (0086)-87755766-8211; mail: chenjh45@mail.sysu.edu.cn and chenchqi@mail.sysu.edu.cn \\ (c) The author(s). This is an open access article distributed under the terms of the Creative Commons Attribution License (https://creativecommons.org/licenses/by/4.0/). \\ See http://ivyspring.com/terms for full terms and conditions.
}

Received: 2020.03.29; Accepted: 2020.06.24; Published: 2020.07.09

\begin{abstract}
Objective: Synchronic colorectal peritoneal carcinomatosis (SCRPC) was recognized as a predictor of poor prognosis. The aim of this study was to investigate the role of neutrophil-to-lymphocyte ratio (NLR), platelet-to-lymphocyte ratio (PLR) and systemic immune-inflammation index (SII) on the survival outcome, which might help determine the treatment management of SCRPC patients.

Methods: A total of 103 SCRPC patients following cytoreduction surgery (CRS) and systematic chemotherapy (CT) between 1997 and 2013 in the First Affiliated Hospital of Sun Yat-sen University were retrospectively analyzed. The comparison of the clinicopathological variables and systematic inflammatory biomarkers, including NLR, PLR and SII, was performed by Chi-test and Cox regression analysis. According to the results of multivariate analysis, a prognostic nomogram was generated, and its prediction ability was measured by the concordance index (C-index). The survival curves were generated using the Kaplan-Meier method and survival comparison between groups was conducted via the log-rank test.
\end{abstract}

Results: Univariate analysis revealed that elevated NLR, PLR and SIl were significantly correlate with worse survival outcome. Only low SII value was recognized as an independent favorable prognostic factor for overall survival $(H R=1.772,95 \% \mathrm{Cl}=1.015-3.095, P=0.044)$, except for $N L R$ and PLR. The nomogram could perform well in the prediction of overall survival in SCRPC patients (c-index 0.782). Moreover, SII had strong prognostic discriminatory ability to predict survival outcome for the patients receiving completeness of cytoreduction score (CCR) $0 / 1$ or CCR2/3, rather than NLR and PLR.

Conclusions: SIl was a better inflammation factor to predict the outcomes of SCRPC patients receiving CRS and systematic CT. Low SIl value was the most favorable factor benefiting from different level of CRS and it was useful for determining the appropriate treatment strategy for SCRPC patients.

Key words: Synchronic colorectal peritoneal carcinomatosis; neutrophil-to-lymphocyte ratio; platelet-to-lymphocyte ratio; systemic immune-inflammation index; prognosis.

\section{Introduction}

Approximately $5-10 \%$ of colorectal cancer (CRC) patients have synchronic peritoneal metastasis

(PM) ${ }^{[1,2]}$ and PM is the second most frequent CRC metastatic site at the time of initial diagnosis [3]. 
Synchronic colorectal peritoneal carcinomatosis (SCRPC) is regarded as a poor prognostic factor for CRC patients [4]. Franko [4] analyzed the database of Analysis and Research in Cancers of the Digestive System (ARCAD) and reported that patients with isolated peritoneal metastasis had worse median survival times (16.3 months) than those with liver (19.1 months), lung (24.6 months), or lymph node (19.4 months) only respectively. Moreover the tumor biological behavior, survival outcome and therapeutic effect of isolated PM origined from CRC significantly differed from other subsets of metastatic colorectal cancer (mCRC) [6, 7]. It was important to find out appropriate prognostic markers to predict survival outcome and guide surgeons to choose optimal treatment strategies for SCRPC patients.

Accumulating evidence supported that the interplay between local immune response and systemic inflammation may play a fundamental role in the development and progression of various cancers [8-10], including CRC [11, 12]. The levels of neutrophil, lymphocyte and platelet via the complete blood count may shed light on the systemic inflammatory response. However, the inflammatory parameters alone may be easily influenced by other factors, thus the joined tools of inflammatory indices, such as neutrophil to lymphocyte ratio (NLR), platelet to lymphocyte ratio (PLR), may be theoretically more reliable and have potentials as powerful candidates to evaluate the host immune status. Likewise, growing evidence supported a strong close relationship between elevated NLR and PLR and worse survival outcome in CRC patients [13, 14]. Systemic immune-inflammation index (SII) was a novel systemic inflammatory index, based on neutrophil, platelet and lymphocyte counts. We previously reported that SII can provided more promising prognostic information than NLR and PLR in CRC patients following radical surgery ${ }^{[15]}$. However, to the best of our knowledge, the prognostic significance of these inflammatory indices, like NLR, PLR and SII, for the SCRPC cases following cytoreduction surgery (CRS) and adjuvant systemic chemotherapy still has not been well studied.

Hence this study aimed to evaluate and compare the prognostic value of different inflammatory indices in SCRPC patients who underwent CRS and systemic chemotherapy and to select an optical inflammatory factor to reflect survival outcome.

\section{Methods}

\section{Study population}

This study was retrospectively analyzed from the database of the Gastrointestinal Surgical Center of the First Affiliated Hospital of Sun Yat-sen University between January 1997 and December 2013. The inclusion criteria were listed below:(1) pathological diagnosis of colorectal adenocarcinoma with PM; (2) patients received cytoreductive surgery and systematic chemotherapy; (3) isolated PM was confirmed according to preoperative findings and intraoperative exploration; (4) patients with complete perioperative clinicopathological, laboratory records and therapeutic interventions. Patients with following criteria were excluded: (1) preoperative anti-tumor or anti-immune treatment, such as chemotherapy, radiotherapy, chemoradiotherapy, immunotherapy; (2) incomplete preoperative clinicopathological and laboratory data or loss of follow-up data; (3) concurrent cancers, recurrent disease or a history of other malignancies within the preceding 5 years; (4) clinical or radiological evidence of inflammatory, infectious or other autoimmune diseases; (5) peritoneal involvement was not confirmed by the pathological examination. At last, 103 SCRPC cases were included in this study. No one had intraoperative chemotherapy or postoperative hyperthermic intraperitoneal chemoperfusion (HIPEC). Patients received postoperative chemotherapy based on a 5-fluorouracil and platinum regimen.Postoperative systemic chemotherapy. The data of the patient's last contact was used as the end of follow-up in all censored patients. Follow-up was updated until December 2018. The study was approved by the independent Ethics Committees of the First Affiliated Hospital of Sun Yat-sen University and was performed in accordance with the ethical standards of the World Medical Association Declaration of Helsinki.

\section{Data collection}

The collected clinical and pathological data in this study was listed as follows: age, gender, preoperative blood test (NLR and PLR), intraoperative blood transfusion, serum carcinoembryonic antigen (CEA), location of primary tumor, histological type, depth of tumor invasion (T stage), lymph node involvement ( $\mathrm{N}$ stage), extend of PM, completeness of CRS.

All the blood tests were performed within 7 days before surgery. NLR and PLR were calculated as the radio of absolute neutrophils counts and platelet counts divided by absolute lymphocyte counts. SII was calculated using the following formula: $\mathrm{SII}=\mathrm{P}^{*} \mathrm{~N} / \mathrm{L}$. where $\mathrm{P}, \mathrm{N}$, and $\mathrm{L}$ refer to the peripheral platelet, neutrophils, and lymphocyte counts, respectively.

Location of primary tumor was classified into two categories: right-colon and left-colon. Right-colon 
included cecum, ascending colon and transverse colon. Left-colon included descending colon, sigmoid colon and rectum. $\mathrm{T}$ stage and $\mathrm{N}$ stage were reclassified according to the $8^{\text {th }}$ edition of $\mathrm{TNM}$ classification.

A complete abdominal cavity exploration was performed to evaluate the extent of peritoneal seeding, which was recorded as PCI score [16]. Eligible patients were divided into two groups based on the extent of peritoneal carcinomatosis: limited ( $\mathrm{PCI} \leq 13)$ and extended (PCI>13).

Completeness of cytoreduction score (CCR) was marked at the end of CRS to assess the volume of residual disease and classified into four categories: CCR0 indicated no macroscopic residual cancer remained; CCR1 indicated no residual nodule larger than $5 \mathrm{~mm}$ in diameter remained; CCR2 indicated the residual nodule ranged from $5 \mathrm{~mm}$ to $2.5 \mathrm{~cm}$ in diameter remained; CCR3 indicated the residual nodule larger than $2.5 \mathrm{~cm}$ remained. We divided patients into two groups: CCR0/1 and CCR2/3.

\section{Statistical analysis}

All the analyses were conducted using SPSS software 20.0 (SPSS Inc., Chicago, USA). Receiver operating characteristic (ROC) curves with Youden Index correction [maximum (sensitivity+specificity-1)] were used to calculate the optimal prognostic cutoff value of NLR, PLR and SII. Chi-square tests were used to analyze the relationship between NLR, PLR, SII and clinicopathological parameters. The survival curves were drawn using the Kaplan-Meier method and survival comparison between groups was conducted via the log-rank test. Only the variables which were significant prognostic parameters in the univariate Cox's proportional hazards model were included in the multivariate analysis to identify independent prognostic factors for SCRPC patients. The nomogram was explored by the "rms" package of R v3.0.0 software (Institute for Statistics and Mathematics, Vienna, Austria) and the concordance index (C-index) was calculated to predict the performance of the established nomogram model. Statistical significance was established at $p<0.05$ for all tests.

\section{Results}

\section{ROC curve analysis of inflammatory indices of SCRPC patients}

ROC curve analysis was used to determine the best cut-off values of NLR, PLR, SII of 2.6 (AUC $=0.662, \quad 95 \% \quad$ CI: $55.8 \%-76.6 \%$, Se $=49.4 \%$, $\mathrm{Sp}=90.0 \%), 144 \quad(\mathrm{AUC}=0.755,95 \% \mathrm{CI}: 64.5 \%-86.5 \%$, $\mathrm{Se}=56.6 \%, \mathrm{Sp}=80.0 \%), 410 \quad(\mathrm{AUC}=0.755, \quad 95 \% \quad \mathrm{CI}$ :
64.5\%-86.5\%, Se=75.9\%, Sp=60.0\%). Among 103 included patients, NLR $\geq 2.6, \mathrm{PLR} \geq 144, \mathrm{SII} \geq 410$ were considered as high groups based on the above cut-off results (Fig 1 \&Table 1).

\section{Clinicopathological Characteristics of SCRPC patients}

Based on the cut-off results above, included patients were divided into two groups: 62 and 41 had low and high NLR values, 50 and 53 had low and high PLR values, 32 and 71 had low and high SII values. Baseline clinicopathological parameters of SCRPC cases following CRS and systematic chemotherapy were shown in Table 2. There was no correlation between high NLR, PLR, SII value and poor histological type, elevated CEA level, larger tumor size, tumor invasion and lymph node involvement, which all considered as negative prognostic factors for CRC patients. The tumor in the high NLR and SII group inclined to locate in the right-hemicolon, rather than tumor in the high PLR group. Among eligible SCRPC patients, larger proportions of extended peritoneal carcinomatosis were found in high NLR $(48.8 \%$ vs $14.5 \%, p<0.001)$, PLR $(49.1 \%$ vs $6.0 \%$, $p<0.001)$ and SII $(38.0 \%$ vs $6.2 \%, p=0.001)$ groups respectively. Moreover, in the cases with low NLR, PLR and SII value, the ratio of patients following CCR0/1 surgery was even greater, increasing from $46.3 \%$ to $79.0 \%, 45.3 \%$ to $88.0 \%, 56.3 \%$ to $87.5 \%$, respectively.

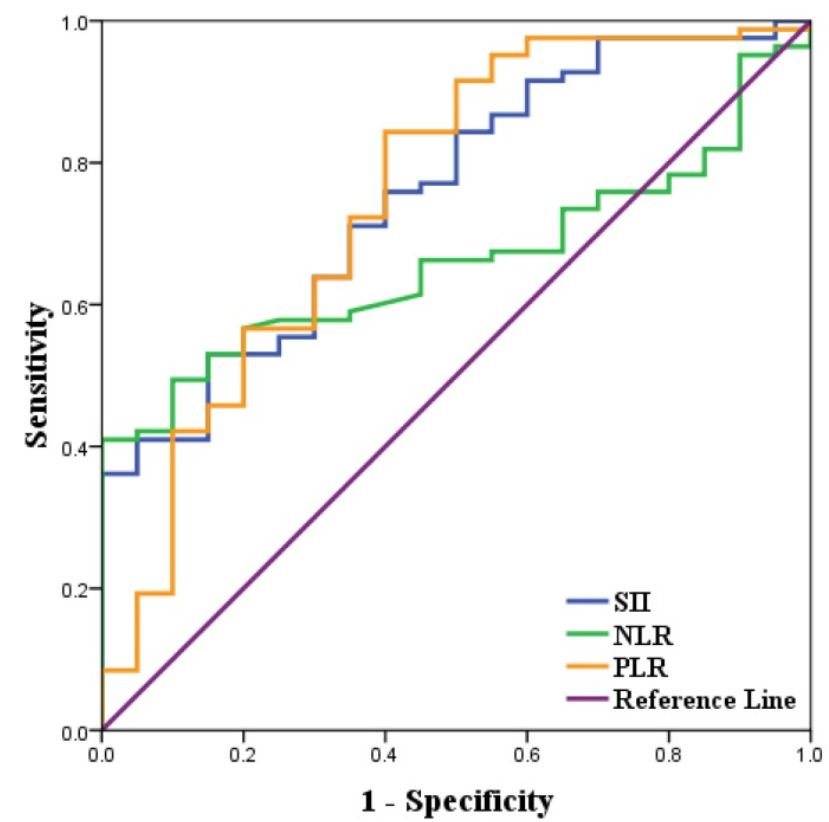

Figure 1. Receiver operating characteristics curve analysis of NLR, PLR, and SII in SCRPC patients following CRS and systemic chemotherapy. 
Table 1. Baseline SCRPC patient characteristics based on.

\begin{tabular}{|c|c|c|c|c|c|c|c|c|c|c|c|}
\hline & & \multirow[t]{2}{*}{ Cases } & \multicolumn{2}{|l|}{ NLR } & \multirow[t]{2}{*}{$P$ value } & \multicolumn{2}{|l|}{ PLR } & \multirow[t]{2}{*}{$P$ value } & \multicolumn{2}{|l|}{ SII } & \multirow[t]{2}{*}{$P$ value } \\
\hline & & & NLR $<2.6$ & NLR $\geq 2.6$ & & PLR $<144$ & PLR $\geq 144$ & & $\mathrm{SII}<410$ & $\mathrm{SII} \geq 410$ & \\
\hline \multicolumn{5}{|l|}{ Age(years) } & \multicolumn{3}{|l|}{0.183} & \multicolumn{3}{|l|}{0.131} & \multirow[t]{3}{*}{0.266} \\
\hline$\leqq 60$ & & 56 & $37(59.7 \%)$ & $19(46.3 \%)$ & & $31(62.0 \%)$ & $25(47.2 \%)$ & & $20(62.5 \%)$ & $36(50.7 \%)$ & \\
\hline$>60$ & & 47 & $25(40.3 \%)$ & $22(53.7 \%)$ & & $19(38.0 \%)$ & $28(52.8 \%)$ & & $12(37.5 \%)$ & $35(49.3 \%)$ & \\
\hline \multicolumn{5}{|l|}{ Gender } & \multicolumn{3}{|l|}{0.260} & 0.151 & & & \multirow[t]{3}{*}{0.934} \\
\hline Male & & 67 & $43(69.4 \%)$ & $24(58.5 \%)$ & & $36(72.0 \%)$ & $31(58.5 \%)$ & & $21(65.6 \%)$ & $46(64.8 \%)$ & \\
\hline Female & & 36 & $19(30.6 \%)$ & $17(41.5 \%)$ & & $14(28.0 \%)$ & $22(41.5 \%)$ & & $11(34.4 \%)$ & $25(35.2 \%)$ & \\
\hline \multicolumn{5}{|l|}{ Tumor location } & \multicolumn{3}{|l|}{$<0.001$} & 0.110 & & & \multirow[t]{3}{*}{0.007} \\
\hline Right-sided & & 39 & $15(24.2 \%)$ & $24(58.5 \%)$ & & $15(30.0 \%)$ & $24(45.3 \%)$ & & $6(18.8 \%)$ & $33(46.5 \%)$ & \\
\hline Left-sided & & 64 & $47(75.8 \%)$ & $17(41.5 \%)$ & & $35(70.0 \%)$ & $29(54.7 \%)$ & & $26(81.2 \%)$ & $38(53.5 \%)$ & \\
\hline \multicolumn{5}{|l|}{ Histological type } & \multicolumn{3}{|l|}{0.068} & 0.347 & & & \multirow[t]{3}{*}{0.250} \\
\hline Well-differentiated & & 59 & $40(64.5 \%)$ & $19(46.3 \%)$ & & $31(62.0 \%)$ & $28(52.8 \%)$ & & $21(65.6 \%)$ & $38(53.5 \%)$ & \\
\hline Poor-differentiated & & 44 & $22(35.5 \%)$ & $22(53.7 \%)$ & & $19(38.0 \%)$ & $25(47.2 \%)$ & & $11(34.4 \%)$ & $33(46.5 \%)$ & \\
\hline \multicolumn{5}{|l|}{ CEA level } & \multicolumn{3}{|l|}{0.544} & 0.632 & & & \multirow[t]{3}{*}{0.449} \\
\hline$\leqq 5 \mathrm{ng} / \mathrm{ml}$ & & 49 & $31(50.0 \%)$ & $18(43.9 \%)$ & & $25(50.0 \%)$ & $24(45.3 \%)$ & & $17(53.1 \%)$ & $32(45.1 \%)$ & \\
\hline$>5 \mathrm{ng} / \mathrm{ml}$ & & 54 & $31(50.0 \%)$ & $23(56.1 \%)$ & & $25(50.0 \%)$ & $29(54.7 \%)$ & & $15(46.9 \%)$ & $39(54.9 \%)$ & \\
\hline \multicolumn{5}{|l|}{ Tumor size } & \multicolumn{3}{|l|}{0.192} & 0.554 & & & \multirow[t]{3}{*}{0.159} \\
\hline$\leqq 5 \mathrm{~cm}$ & & 38 & $26(41.9 \%)$ & $12(29.3 \%)$ & & $17(34.0 \%)$ & $21(39.6 \%)$ & & $15(46.9 \%)$ & $23(32.4 \%)$ & \\
\hline$>5 \mathrm{~cm}$ & & 65 & $36(58.1 \%)$ & $29(70.7 \%)$ & & $33(66.0 \%)$ & $32(60.4 \%)$ & & $17(53.1 \%)$ & $48(67.6 \%)$ & \\
\hline $\begin{array}{l}\text { Intraoperative } \\
\text { transfusion }\end{array}$ & blood & & & & 0.304 & & & 0.705 & & & 0.959 \\
\hline No & & 39 & $21(33.9 \%)$ & $18(43.9 \%)$ & & $18(36.0 \%)$ & $21(39.6 \%)$ & & $12(37.5 \%)$ & $27(38.0 \%)$ & \\
\hline Yes & & 64 & $41(66.1 \%)$ & $23(56.1 \%)$ & & $32(64.0 \%)$ & $32(60.4 \%)$ & & $20(62.5 \%)$ & $44(62.0 \%)$ & \\
\hline T stage & & & & & 0.958 & & & 0.317 & & & 0.598 \\
\hline $\mathrm{T} 3$ & & 38 & $23(37.1 \%)$ & $15(36.6 \%)$ & & $16(32.0 \%)$ & $22(41.5 \%)$ & & $13(40.6 \%)$ & $25(35.2 \%)$ & \\
\hline $\mathrm{T} 4$ & & 65 & $39(62.9 \%)$ & $26(63.4 \%)$ & & $34(68.0 \%)$ & $31(58.5 \%)$ & & $19(59.4 \%)$ & $46(64.8 \%)$ & \\
\hline Lymph node metas & & & & & 0.677 & & & 0.691 & & & 0.462 \\
\hline $\mathrm{N}^{0}$ & & 17 & $11(17.7 \%)$ & $6(14.6 \%)$ & & $9(18.0 \%)$ & $8(15.1 \%)$ & & $4(12.5 \%)$ & $13(18.3 \%)$ & \\
\hline $\mathrm{N}^{+}$ & & 86 & $51(82.3 \%)$ & $35(85.4 \%)$ & & $41(82.0 \%)$ & $45(84.9 \%)$ & & $28(87.5 \%)$ & $58(81.7 \%)$ & \\
\hline PM level & & & & & 0.001 & & & $<0.001$ & & & 0.001 \\
\hline limited & & 74 & $52(83.9 \%)$ & $22(53.7 \%)$ & & $47(94.0 \%)$ & $27(50.9 \%)$ & & $30(93.8 \%)$ & $44(62.0 \%)$ & \\
\hline extended & & 29 & $10(16.1 \%)$ & $19(46.3 \%)$ & & $3(6.0 \%)$ & $26(49.1 \%)$ & & $2(6.2 \%)$ & $27(38.0 \%)$ & \\
\hline CCR score & & & & & 0.001 & & & $<0.001$ & & & 0.002 \\
\hline CCR0/1 & & 68 & $49(79.0 \%)$ & $19(46.3 \%)$ & & $44(88.0 \%)$ & $24(45.3 \%)$ & & $28(87.5 \%)$ & $40(56.3 \%)$ & \\
\hline CCR $2 / 3$ & & 35 & $13(21.0 \%)$ & $22(53.7 \%)$ & & $6(12.0 \%)$ & $29(54.7 \%)$ & & $4(12.5 \%)$ & $31(43.7 \%)$ & \\
\hline
\end{tabular}

A

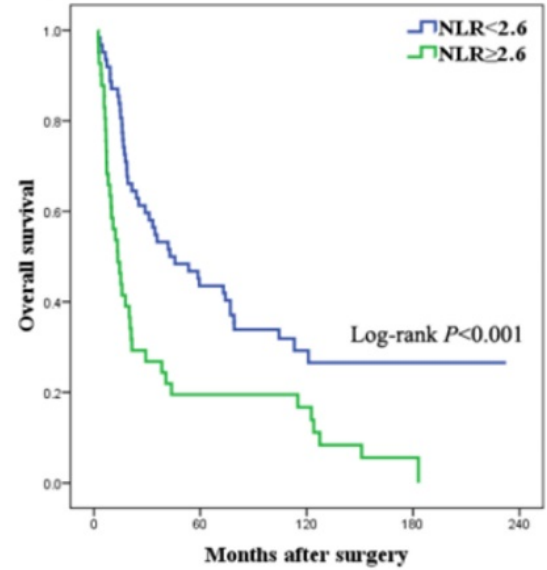

B

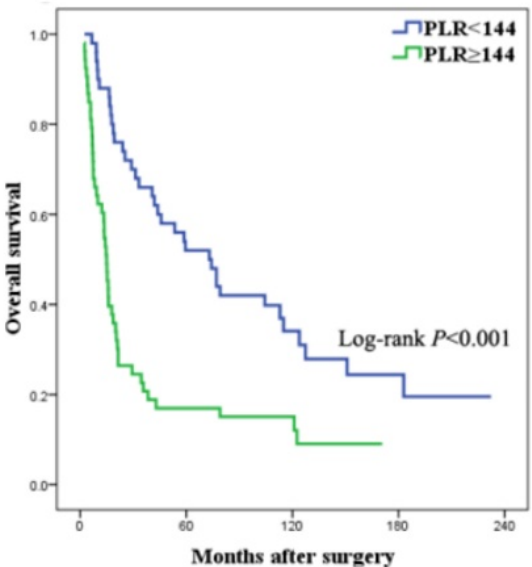

C

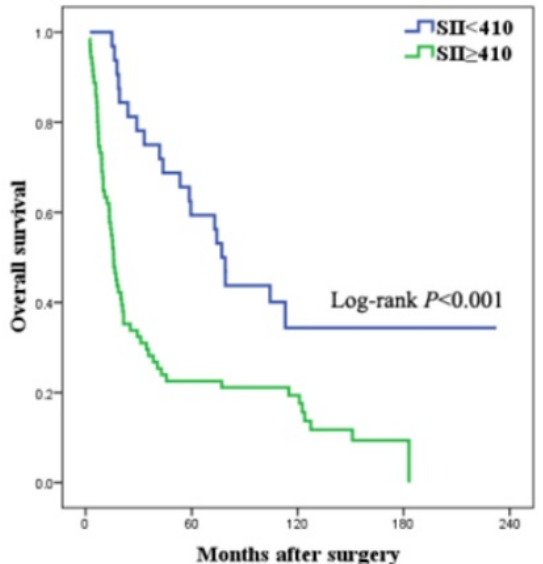

Figure 2. Survival curves of SCRPC patients following CRS and systemic chemotherapy in different NLR (a), PLR (b), and SII (c) group.

\section{Overall survival}

The mean follow-up time for this study was 55.4 months. In this cohort, the median OS was 23.8 months, while 2, 5, 10-years overall survival rate was $50.0 \%, 34.0 \%$ and $24.2 \%$, respectively.

The survival data was listed in Table 2. The median survival time of patients in low NLR, PLR and SII group was 44.0, 74.4, 79.2 months and it in high NLR, PLR and SII group was 14.7, 16.1, 17.7 months respectively. Using log-rank test, the low NLR, PLR and SII group statistically had better long-term overall survival outcome than the high NLR, PLR and SII group respectively (Figure 2a-c).

Among all the included factors, univariate analysis showed that age, tumor location, histological type, CEA level, $\mathrm{N}$ stage, extent of peritoneal carcinomatosis, completeness of CRS, NLR value, PLR value and SII value were associated with prognosis, whereas age, gender, $\mathrm{T}$ stage, intraoperative blood transfusion were not significantly associated with OS (Table 3). According to the multivariate analysis, only 
tumor located in the right-hemicolon, elevated CEA level, extended PM, CCR0/1 and high SII value were independent factors associated with a worse prognosis (Table 3), rather than NRL value and PRL value.

To further predict the survival of SCRPC patients after CRS and systematic CT, all the significant independent risk prognostic factors of the primary cohort were integrated in the nomogram to predict the 2-, 5- and 10-year survival using multivariate Cox regression model analysis (Fig. 3). The C-index for OS prediction was 0.782, which closed corresponded to the actual survival.

\section{Overall survival stratified by completeness of CRS}

Among the patients following CCR 0/1 surgery, age, tumor size, T stage, PM level, intraoperative blood transfusion and SII value were associated with overall survival (Table 4). Of these factors, multivariate analysis still showed that low SII value remained a strong prognostic factor for improved overall survival, not NLR and PLR. Survival curve among different levels of NLR, PLR and SII was shown in Figure 4a-c and survival data was listed in Table 2.

Of the $35(34.0 \%)$ patients underwent CCR 2/3 surgery, the survival curves (Fig. $4 \mathrm{~d}$-f) were drawn to describe the relationship between OS and NLR, PLR and SII value. Following prognostic factors were significant in univariate analysis: tumor location, CEA level, PM level, PLR value and SII value. Multivariate analysis was further performed for significant factors in univariate test. Only low CEA level and low SII value were regarded as favorable prognostic factor in this cohort (Table 5).

Table 2. Survival rate of OS for SCRPC patients.

\begin{tabular}{|c|c|c|c|c|c|c|c|}
\hline & \multicolumn{3}{|c|}{ Low value } & \multicolumn{3}{|c|}{ High value } & \multirow{2}{*}{$\begin{array}{l}P \\
\text { value }\end{array}$} \\
\hline & $2 \mathrm{ysr}$ & $5 y s r$ & $10 y s r$ & $2 y s r$ & $5 y s r$ & $10 y s r$ & \\
\hline \multicolumn{8}{|c|}{ Whole } \\
\hline NLR & $62.9 \%$ & $43.5 \%$ & $29.3 \%$ & $29.3 \%$ & $19.5 \%$ & $16.7 \%$ & $<0.001$ \\
\hline PLR & $74.0 \%$ & $52.0 \%$ & $34.2 \%$ & $26.4 \%$ & $17.0 \%$ & $15.1 \%$ & $<0.001$ \\
\hline SII & $81.3 \%$ & $59.4 \%$ & $34.6 \%$ & $35.2 \%$ & $22.5 \%$ & $19.4 \%$ & $<0.001$ \\
\hline \multicolumn{8}{|c|}{ CCR0/1 } \\
\hline NLR & $77.6 \%$ & $55.1 \%$ & $37.0 \%$ & $57.9 \%$ & $42.1 \%$ & $36.1 \%$ & 0.134 \\
\hline PLR & $81.8 \%$ & $59.1 \%$ & $38.9 \%$ & $54.2 \%$ & $37.5 \%$ & $33.3 \%$ & 0.141 \\
\hline SII & $60.0 \%$ & $40.0 \%$ & $34.4 \%$ & $89.3 \%$ & $67.9 \%$ & $39.6 \%$ & 0.026 \\
\hline \multicolumn{8}{|c|}{ CCR2/3 } \\
\hline NLR & $7.7 \%$ & $0.0 \%$ & $0.0 \%$ & $4.5 \%$ & $0.0 \%$ & $0.0 \%$ & 0.178 \\
\hline PLR & $16.7 \%$ & $0.0 \%$ & $0.0 \%$ & $3.4 \%$ & $0.0 \%$ & $0.0 \%$ & 0.047 \\
\hline SII & $25.0 \%$ & $0.0 \%$ & $0.0 \%$ & $3.2 \%$ & $0.0 \%$ & $0.0 \%$ & 0.033 \\
\hline
\end{tabular}

Table 3. Univariate and multivariate analyses of factors affecting CRC patients with peritoneal metastases after palliative primary tumor resection and cytoreductive surgery.

\begin{tabular}{|c|c|c|c|c|c|c|c|c|}
\hline & \multicolumn{4}{|c|}{ Univariate analysis } & \multicolumn{4}{|c|}{ Multivariate analysis } \\
\hline & $\chi^{2}$ value & HR & $95 \% \mathrm{CI}$ & $P$ value & $\chi^{2}$ value & HR & $95 \% \mathrm{CI}$ & $P$ value \\
\hline Age $(\leqq 60 y$ vs $>60 y)$ & 3.575 & - & - & 0.059 & & & & \\
\hline Gender (male vs female) & 0.712 & - & - & 0.399 & & & & \\
\hline Tumor location (left vs right) & 4.615 & 0.619 & $0.400-0.959$ & 0.032 & 4.019 & 0.618 & $0.385-0.989$ & 0.045 \\
\hline Histological type (well vs poor-differentiated) & 3.957 & 1.551 & $1.006-2.390$ & 0.047 & & & & \\
\hline Tumor size $(\leqq 5 \mathrm{~cm}$ vs $>5 \mathrm{~cm})$ & 0.801 & - & - & 0.371 & & & & \\
\hline CEA level $(\leqq 5 \mathrm{ng} / \mathrm{ml}$ vs $>5 \mathrm{ng} / \mathrm{ml})$ & 4.661 & 1.614 & $1.045-2.493$ & 0.031 & 6.959 & 1.844 & $1.170-2.904$ & 0.008 \\
\hline T stage $\left(\mathrm{T}_{3}\right.$ vs $\left.\mathrm{T}_{4}\right)$ & 1.551 & - & - & 0.213 & & & & \\
\hline Lymph node metastasis $\left(\mathrm{N}_{0}\right.$ vs $\left.\mathrm{N}_{+}\right)$ & 4.487 & 1.995 & $1.053-3.781$ & 0.034 & & & & \\
\hline PM level (limited vs extended) & 46.668 & 3.684 & $2.534-5.356$ & $<0.001$ & 4.530 & 2.340 & $1.070-5.118$ & 0.033 \\
\hline Completeness of CRS (CRS0/ 1 vs CRS2/3) & 53.634 & 6.982 & $4.151-11.745$ & $<0.001$ & 15.347 & 4.471 & 2.114-9.459 & $<0.001$ \\
\hline Intraoperative blood transfusion (no vs yes) & 3.571 & - & - & 0.059 & & & & \\
\hline NLR value (low vs high) & 12.241 & 2.174 & $1.407-3.359$ & $<0.001$ & & & & \\
\hline PLR value (low vs high) & 17.535 & 2.593 & $1.660-4.050$ & $<0.001$ & & & & \\
\hline SII value (low vs high) & 14.057 & 2.651 & $1.592-4.413$ & $<0.001$ & 4.048 & 1.772 & $1.015-3.095$ & 0.044 \\
\hline
\end{tabular}

Table 4. Univariate and multivariate analysis of factors affecting SCRPC patients following CCRO/1.

\begin{tabular}{|c|c|c|c|c|c|c|c|c|}
\hline & \multicolumn{4}{|c|}{ Univariate analysis } & \multicolumn{4}{|c|}{ Multivariate analysis } \\
\hline & $\chi^{2}$ value & HR & $95 \% \mathrm{CI}$ & $P$ value & $\chi^{2}$ value & HR & $95 \% \mathrm{CI}$ & $P$ value \\
\hline $\begin{array}{l}\text { Age } \\
(\leqq 60 \mathrm{y} \text { vs }>60 \mathrm{y})\end{array}$ & 3.892 & 1.784 & $1.004-3.171$ & 0.049 & 8.087 & 2.473 & $1.325-4.614$ & 0.004 \\
\hline $\begin{array}{l}\text { Gender } \\
\text { (male vs female) }\end{array}$ & 0.415 & - & - & 0.519 & & & & \\
\hline $\begin{array}{l}\text { Tumor location } \\
\text { (left vs right) }\end{array}$ & 2.967 & - & - & 0.085 & & & & \\
\hline $\begin{array}{l}\text { Histological type } \\
\text { (well vs poor-differentiated) }\end{array}$ & 2.387 & - & - & 0.122 & & & & \\
\hline $\begin{array}{l}\text { Tumor size } \\
(\leqq 5 \mathrm{~cm} \text { vs }>5 \mathrm{~cm})\end{array}$ & 4.570 & 0.531 & $0.297-0.949$ & 0.033 & & & & \\
\hline $\begin{array}{l}\text { CEA level } \\
(\leqq 5 \mathrm{ng} / \mathrm{ml} \text { vs }>5 \mathrm{ng} / \mathrm{ml})\end{array}$ & 1.743 & - & - & 0.187 & & & & \\
\hline $\begin{array}{l}\text { T stage } \\
\left(\mathrm{T}_{3} \mathrm{Vs}_{4}\right)\end{array}$ & 6.805 & 0.461 & $0.258-0.825$ & 0.009 & 6.287 & 0.463 & $0.254-0.845$ & 0.012 \\
\hline $\begin{array}{l}\text { Lymph node metastasis } \\
\left(\mathrm{N}_{0} \text { vs } \mathrm{N}_{+}\right)\end{array}$ & 3.141 & - & - & 0.076 & & & & \\
\hline
\end{tabular}




\begin{tabular}{|c|c|c|c|c|c|c|c|c|}
\hline & \multicolumn{4}{|c|}{ Univariate analysis } & \multicolumn{4}{|c|}{ Multivariate analysis } \\
\hline & $\chi^{2}$ value & $\mathrm{HR}$ & $95 \% \mathrm{CI}$ & $P$ value & $X^{2}$ value & HR & $95 \% \mathrm{CI}$ & $P$ value \\
\hline $\begin{array}{l}\text { PM level } \\
\text { (limited vs extended) }\end{array}$ & 9.475 & 6.956 & $2.023-23.917$ & 0.002 & 15.190 & 14.081 & $3.724-53.240$ & $<0.001$ \\
\hline $\begin{array}{l}\text { Intraoperative blood transfusion } \\
\text { (no vs yes) }\end{array}$ & 5.879 & 0.485 & $0.270-0.871$ & 0.015 & 5.864 & 0.470 & $0.255-0.866$ & 0.015 \\
\hline $\begin{array}{l}\text { NLR value } \\
\text { (low vs high) }\end{array}$ & 2.246 & - & - & 0.134 & & & & \\
\hline $\begin{array}{l}\text { PLR value } \\
\text { (low vs high) }\end{array}$ & 2.164 & - & - & 0.141 & & & & \\
\hline $\begin{array}{l}\text { SII value } \\
\text { (low vs high) }\end{array}$ & 4.753 & 1.963 & $1.071-3.601$ & 0.029 & 6.502 & 2.277 & $1.210-4.288$ & 0.011 \\
\hline
\end{tabular}

Table 5. Univariate and multivariate analysis of factors affecting SCRPC patients following CCR2/3.

\begin{tabular}{|c|c|c|c|c|c|c|c|c|}
\hline & \multicolumn{4}{|c|}{ Univariate analysis } & \multicolumn{4}{|c|}{ Multivariate analysis } \\
\hline & $\chi^{2}$ value & HR & $95 \% \mathrm{CI}$ & $P$ value & $\chi^{2}$ value & HR & $95 \% \mathrm{CI}$ & $P$ value \\
\hline $\begin{array}{l}\text { Age } \\
(\leqq 60 y \text { vs }>60 y)\end{array}$ & 0.136 & - & - & 0.713 & & & & \\
\hline $\begin{array}{l}\text { Gender } \\
\text { (male vs female) }\end{array}$ & 0.661 & - & - & 0.416 & & & & \\
\hline $\begin{array}{l}\text { Tumor location } \\
\text { (left vs right) }\end{array}$ & 4.611 & 0.446 & $0.213-0.932$ & 0.032 & & & & \\
\hline $\begin{array}{l}\text { Histological type } \\
\text { (well vs poor-differentiated) }\end{array}$ & 1.755 & - & - & 0.185 & & & & \\
\hline $\begin{array}{l}\text { Tumor size } \\
(\leqq 5 \mathrm{~cm} \text { vs }>5 \mathrm{~cm})\end{array}$ & 2.314 & - & - & 0.128 & & & & \\
\hline $\begin{array}{l}\text { CEA level } \\
(\leqq 5 \mathrm{ng} / \mathrm{ml} \text { vs }>5 \mathrm{ng} / \mathrm{ml})\end{array}$ & 5.342 & 2.307 & $1.135-4.687$ & 0.021 & 6.123 & 2.484 & $1.208-5.107$ & 0.013 \\
\hline $\begin{array}{l}\text { T stage } \\
\left(\mathrm{T}_{3} \mathrm{vs}_{4}\right)\end{array}$ & 0.166 & - & - & 0.684 & & & & \\
\hline $\begin{array}{l}\text { Lymph node metastasis } \\
\left(\mathrm{N}_{0} \text { vs } \mathrm{N}_{+}\right)\end{array}$ & 1.154 & - & - & 0.283 & & & & \\
\hline $\begin{array}{l}\text { PM level } \\
\text { (limited vs extended) }\end{array}$ & 4.758 & 2.456 & $1.095-5.505$ & 0.029 & & & & \\
\hline $\begin{array}{l}\text { Intraoperative blood transfusion } \\
\text { (no vs yes) }\end{array}$ & 1.187 & - & - & 0.276 & & & & \\
\hline $\begin{array}{l}\text { NLR value } \\
\text { (low vs high) }\end{array}$ & 1.1779 & - & - & 0.182 & & & & \\
\hline $\begin{array}{l}\text { PLR value } \\
\text { (low vs high) }\end{array}$ & 3.626 & 2.547 & $0.973-6.668$ & 0.057 & & & & \\
\hline $\begin{array}{l}\text { SII value } \\
\text { (low vs high) }\end{array}$ & 3.952 & 3.388 & $1.017-11.287$ & 0.047 & 4.495 & 3.721 & $1.104-12.535$ & 0.034 \\
\hline
\end{tabular}

Points

$\begin{array}{lllllllllll}0 & 10 & 20 & 30 & 40 & 50 & 60 & 70 & 80 & 90 & 100\end{array}$

tumor.location

CCR.score

CEAlevel

Sllgroup

$\mathrm{PCl}$ score

Total Points

Linear Predictor

2-year survival

5-year survival

10-year survival
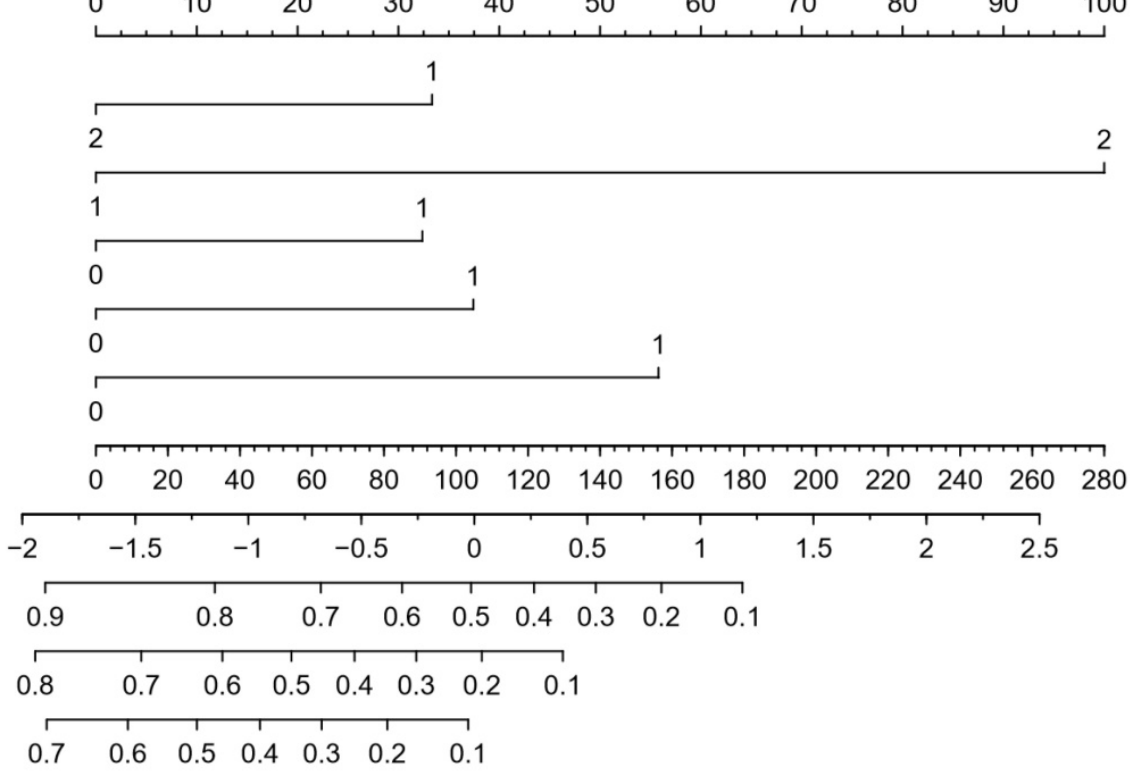

Figure 3. Nomogram of predicting 2-, 5- and 10-year survival for SCRPC patients after CRS and systematic CT. Tumor location: 1, right semicolon; 2 , left semicolon; CCR score: 1, CCR0/1; 2, CCR2/3; CEA level: $0, \leqq 5 \mathrm{ng} / \mathrm{ml} ; 1,>5 \mathrm{ng} / \mathrm{ml}$; SIl group: 0, SIl<410; 1, SII $\geq 410$; PM level: 0, limited; 1, extended. 
A

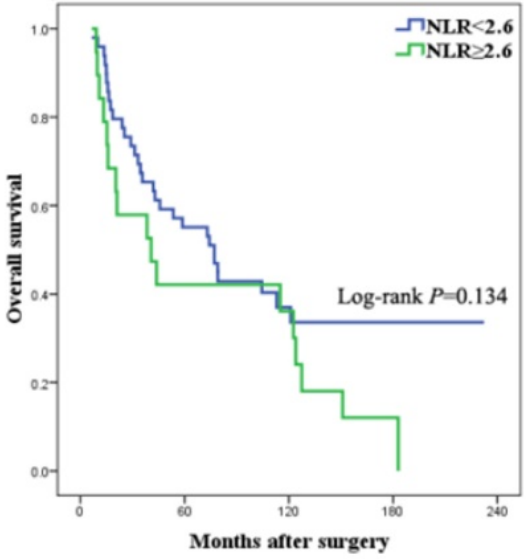

D

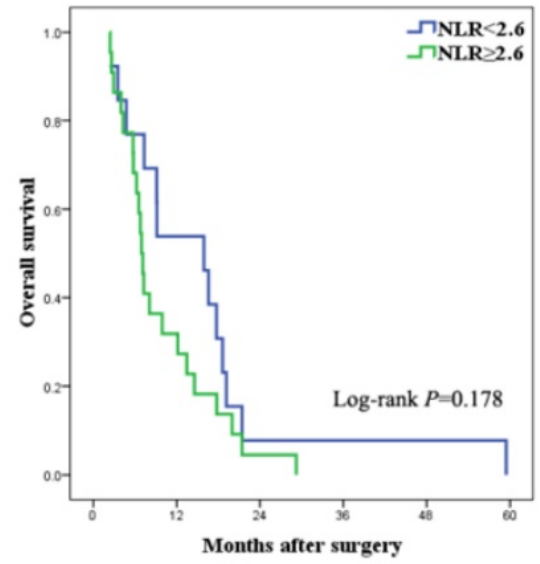

B

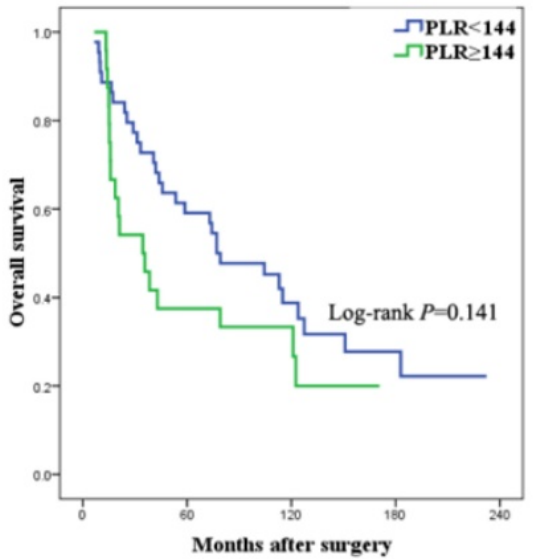

E

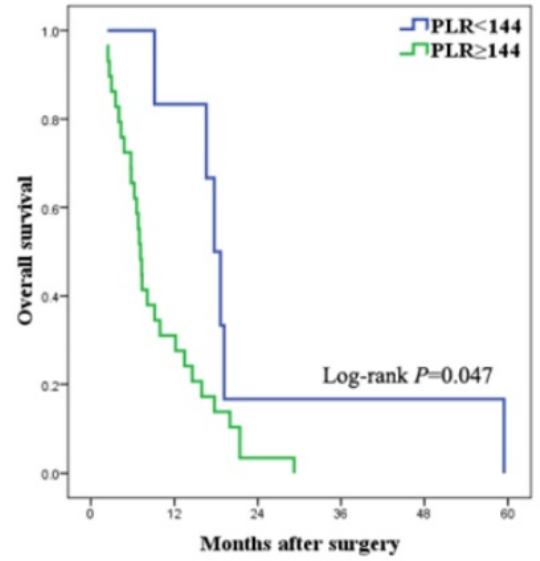

C

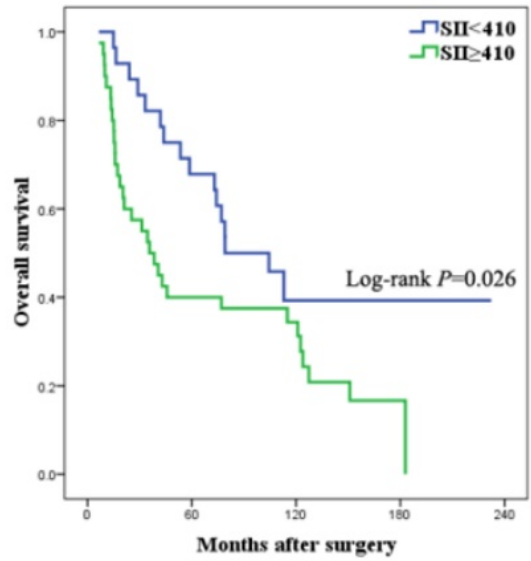

F

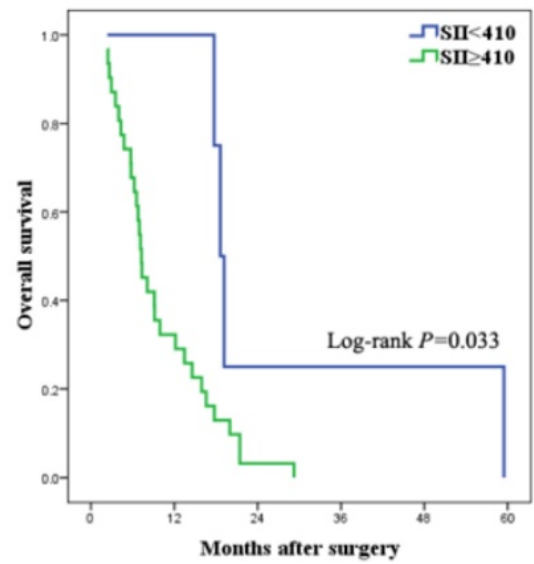

Figure 4. Survival curves of SCRPC patients following different levels of CRS. (a-c) SCRPC patients following CCR 0/1 surgery. a. high vs low NLR group; b. high vs low PLR group; c. high vs low SII group. (d-f) SCRPC patients following CCR 2/3 surgery. d. high vs low NLR group; e high vs low PLR group; f. high vs low SII group.

\section{Discussion}

To the best of our knowledge, this was the first retrospective study to analyze the prognostic significance of systematic inflammatory biomarkers in CRC patients with PM. Our results indicated that preoperative NLR, PLR and SII value all had the abilities to predict survival outcome in our cohort and SII was the only independent prognostic factor. Moreover, SII can distinguish the survival differences in the SCRPC cases independently of the completeness level of CRS.

Although significant progresses have been made in terms of developing non-invasive prognostic biomarkers for CRC, the clinical appliance of the systematic inflammatory indices were regularly used, with its advantage of inexpensive, reliable, reproducible, robust and convenience ${ }^{[17-19]}$. Among all the systematic inflammatory indices, NLR and PLR were the most commonly used as prognostic biomarkers in CRC, which even had a discriminatory ability superior to other inflammatory biomarkers in resectable CRC [20]. While, SII was only recently introduced. Growing evidence demonstrated that SII has shown optical prognostic power in a variety of malignancies, including bladder cancer [21], non-small cell lung cancer [22], gastric [23], pancreatic cancer [24], and CRC [15]. However, no previous studies have investigated the clinical significance of these systematic inflammatory biomarkers in CRC with peritoneal metastasis. Thus, we were the first to reveal that SCRPC patients following CRS and systematic chemotherapy with elevated NLR or PLR or SII levels at initial diagnosis had poor survival outcome. What's more important, SII was the only independent systematic inflammatory prognostic factor to predict long-term survival outcomes of SCRPC patients, not NLR or PLR, which was similar to in primary CRC patients.

Our study showed that the systematic inflammation biomarkers including NLR, PLR and SII correlated with the survival outcome of SCRPC patients. Although precise prognostic mechanism was not completely understood, strong evidences 
considered that the systematic inflammation may play a critical role in pathogenesis and progression of cancer and predict worse survival outcome, including CRC [25-27]. Neutrophils may produce and secret cytokines, chemokines, and proteases, which promoted cancer cells adhesion and seeding in the peritoneum. Lymphopenia destroyed tumor immune defense by inhibiting cancer cells immune surveillance and blocking cytotoxic cell death, which may produce a favorable tumor microenvironment in the peritoneum for the proliferation, progression and spread of CRC cells [28, 29]. Recent evidence suggested that platelets can release platelet-derived growth factors and pro-angiogenic protein (such as interleukin-6) and act as chemoattractants to promote growth, migration and angiogensis of tumor cells. Moreover, tumor cells can directly interact with platelets to facilitate tumor extravasation and metastatic niche formation [30-32]. As a result, the level of serum platelets was positive correlated with the degrees of inflammatory response. Therefore, the elevated level of neutrophils and platelets can reflect systematic inflammation response and the decline of lymphocytes may be responsible for cellular immune injury or systematic immune surveillance. Hence SII based on the above systematic inflammatory indices can better and more objectively reflect the balance between host inflammation and immune response in cancer patients, which might make SII a better biomaker of predicting survival outcome in SCRPC patients.

Although overall survival of SCRPC patients has increased over time with the development of multidisciplinary management (such as HIPEC, CRS, IPC, chemotherapy) and new target drugs (such as bevacuzimab, cetuximab), the efficacy of treatment for SCRPC was still yet not established. As Prodige 7 trial [33] failed to demonstrate the survival benefit was gained from HIPEC with oxaliplatin to a complete CRS. Most SCRPC patients were accompanied by synchronous hepatic or lung metastases. SCRPC occupied less than $5 \%$ of stage IV CRC cases and had a great tendency to develop metachronous distant metastasis. Hence SCRPC may get a potentially survival improvement from systematic chemotherapy. However, the therapeutic concentration in the peritoneal metastatic nodule following systemic administration of chemotherapy was relatively low, possibly because of the lack of vascularization of PC seeding. Thus, some researchers proposed that SCRPC patients without extraperitoneal disease may get more survival benefit from locoregional treatment, such as complete cytoreduction (Ref??). The median survival time of SCRPC patients who underwent CRS was more than 30 months and 5 year survival rate was nearly $30 \%[34]$, similar to the Stage III CRC patients, but, not all patients got survival benefit from complete cytoreduction. The prognostic value of these treatment was still controversial. Therefore, better and more powerful prognostic factors should be explored to help selecting patients who can benefit of such extensive procedure. The Peritoneal Surface Disease Severity Score (PSDSS) [35] and colorectal peritoneal metastases prognostic surgical score (COMPASS) [36] were reported to had a good discriminative ability to improve patient selection. In this study we firstly focused on the clinical appliances of systematic inflammatory factors to predict survival in these patients. The result in our cohort demonstrated that SII was the only systematic inflammatory index who had a stronger prognostic ability to discriminate survival differences independent of the levels of complete cytoreduction and systematic chemotherapy.

There were still some limitations in this study. First, this was a single-center study and the study population was small, so more patients from other centers will be needed for further external validation. Second, although the systematic inflammatory indices were recorded prospectively, the analysis of this study was undertaken in a retrospective fashion. Also, the study was only focused on the OS of selected SCRPC patients who underwent CRS and systematic chemotherapy, and more prognostic indicator will be included in further studies.

\section{Conclusions}

This was the first report to demonstrate that SII had a stronger prognostic value among SCRPC patients, and SII can provide prognostic value to distinguish survival outcome independently of the level of CRS, rather than NLR and PLR. SII was recommended as the prognostic factors to select appropriate SCRPC patients who can benefit from CRS and systematic chemotherapy. However, prospective studies and the larger number of patients are needed to further validate this finding.

\section{Acknowledgements}

This study was supported by research grants from Financial Disclosure information. This study was supported by research grants from the National Natural Science Foundation of China (No.81702878), Natural Science Foundation of Guangdong Province(2020A1515010214), Basic scientific research funding of Sun Yat sen University (14ykpy58).

\section{Author Contributions}

Qian Yan and Zhai Ertao contributed equally to this study, including study concept and design, 
analysis and interpretation of data, drafting of the manuscript, and critical revision of the manuscript for important intellectual content. Zhang Zhimei, Dai Weigang, Peng Jianjun, participated in the acquisition of data and statistical analysis. Chen Jianhui and Chen Chuangqi supervised the whole study as well as monitored the standard surgical operations. All the authors took part in the surgical treatments for colorectal cancer.

\section{Competing Interests}

The authors have declared that no competing interest exists.

\section{References}

[1] Pinto A, Hobeika C, Philis A, et al. Synchronous liver metastases and peritoneal carcinomatosis from colorectal cancer: Different strategies for curative treatment?. Langenbecks Arch Surg. 2019; 404:477-488.

[2] Marz L, Piso P. Treatment of peritoneal metastases from colorectal cancer. Gastroenterol Rep (Oxf). 2015; 3:298-302.

[3] Klaver CE, Musters GD, Bemelman WA, et al. Adjuvant hyperthermic intraperitoneal chemotherapy (HIPEC) in patients with colon cancer at high risk of peritoneal carcinomatosis; The COLOPEC randomized multicentre trial. BMC CANCER. 2015; 15:428

[4] Glockzin G, Schlitt HJ, Piso P. Therapeutic options for peritoneal metastasis arising from colorectal cancer. World J Gastrointest Pharmacol Ther. 2016; 7:343-352.

[5] Franko J, Shi Q, Meyers JP, et al. Prognosis of patients with peritoneal metastatic colorectal cancer given systemic therapy: An analysis of individual patient data from prospective randomised trials from the Analysis and Research in Cancers of the Digestive System (ARCAD) database. LANCET ONCOL. 2016; 17:1709-1719.

[6] Prasanna T, Karapetis CS, Roder D, et al. The survival outcome of patients with metastatic colorectal cancer based on the site of metastases and the impact of molecular markers and site of primary cancer on metastatic pattern. ACTA ONCOL. 2018; 57:1438-1444

[7] Koumpa FS, Xylas D, Konopka M, et al. Colorectal peritoneal metastases: A systematic review of current and emerging trends in clinical and translational research. Gastroenterol Res Pract. 2019; 2019:5180895.

[8] Padoan A, Plebani M, Basso D. Inflammation and Pancreatic Cancer: Focus on Metabolism, Cytokines, and Immunity. INT J MOL SCI. 2019; 20:676.

[9] Diakos CI, Charles KA, McMillan DC, et al. Cancer-related inflammation and treatment effectiveness. LANCET ONCOL. 2014;15: e493-e503.

[10] Huang H, Liu Q, Zhu L, et al. Prognostic value of preoperative systemic Immune-Inflammation index in patients with cervical cancer. Sci Rep. 2019; 9:3284.

[11] Turner N, Wong HL, Templeton A, et al. Analysis of local chronic inflammatory cell infiltrate combined with systemic inflammation improves prognostication in stage II colon cancer independent of standard clinicopathologic criteria. INT J CANCER. 2016; 138:671-678.

[12] Galizia G, Lieto E, Zamboli A, et al. Neutrophil to lymphocyte ratio is a strong predictor of tumor recurrence in early colon cancers: A propensity score-matched analysis. SURGERY. 2015; 158:112-120.

[13] Stojkovic LM, Pavlovic MA, Stankovic S, et al. Combined diagnostic efficacy of Neutrophil-to-Lymphocyte ratio (NLR), Platelet-to-Lymphocyte ratio (PLR), and mean platelet volume (MPV) as biomarkers of systemic inflammation in the diagnosis of colorectal cancer. DIS MARKERS. 2019; 2019:6036979.

[14] Zhang J, Zhang HY, Li J, et al. The elevated NLR, PLR and PLT may predict the prognosis of patients with colorectal cancer: A systematic review and meta-analysis. Oncotarget. 2017; 8:68837-68846.

[15] Chen JH, Zhai ET, Yuan YJ, et al. Systemic immune-inflammation index for predicting prognosis of colorectal cancer. World J Gastroenterol. 2017; 23:6261-6272.

[16] Jacquet P, Sugarbaker PH. Clinical research methodologies in diagnosis and staging of patients with peritoneal carcinomatosis. Cancer Treat Res. 1996; 82:359-374.

[17] Imamoglu GI, Eren T, Baylan B, et al. May high levels of systemic Immune-Inflammation index and hematologic inflammation markers suggest a further stage in testicular tumours?. UROL INT. 2019; 103:303-310.

[18] Luo H, He L, Zhang $G$, et al. Normal reference intervals of Neutrophil-To-Lymphocyte ratio, Platelet-To-Lymphocyte ratio, Lymphocyte-To-Monocyte ratio, and systemic immune inflammation index in healthy adults: A large Multi-Center study from western china. CLIN LAB. 2019; 65.

[19] Fest J, Ruiter R, Ikram MA, et al. Reference values for white blood-cell-based inflammatory markers in the Rotterdam Study: A population-based prospective cohort study. Sci Rep. 2018; 8:10566.
[20] Song Y, Yang Y, Gao P, et al. The preoperative neutrophil to lymphocyte ratio is a superior indicator of prognosis compared with other inflammatory biomarkers in resectable colorectal cancer. BMC CANCER. 2017; 17:744.

[21] Zhang W, Wang R, Ma W, et al. Systemic immune-inflammation index predicts prognosis of bladder cancer patients after radical cystectomy. Ann Transl Med. 2019; 7:431.

[22] $\mathrm{Xu} \mathrm{F}, \mathrm{Xu} \mathrm{P}$, Cui $\mathrm{W}$, et al. Neutrophil-to-lymphocyte and platelet-to-lymphocyte ratios may aid in identifying patients with non-small cell lung cancer and predicting Tumor-Node-Metastasis stages. ONCOL LETT. 2018; 16:483-490.

[23] Wang K, Diao F, Ye Z, et al. Prognostic value of systemic immune-inflammation index in patients with gastric cancer. CHIN J CANCER. 2017; 36:75.

[24] Aziz MH, Sideras K, Aziz NA, et al. The systemic-immune-inflammation index independently predicts survival and recurrence in resectable pancreatic cancer and its prognostic value depends on bilirubin levels: A retrospective multicenter cohort study. ANN SURG. 2019; 270:139-146.

[25] Karki R, Man SM, Kanneganti TD. Inflammasomes and cancer. CANCER IMMUNOL RES. 2017; 5:94-99.

[26] Mei Z, Liu Y, Liu C, et al. Tumour-infiltrating inflammation and prognosis in colorectal cancer: Systematic review and meta-analysis. Br J Cancer. 2014; 110:1595-1605.

[27] Masson-Lecomte A, Rava M, Real FX, et al. Inflammatory biomarkers and bladder cancer prognosis: A systematic review. EUR UROL. 2014; 66:1078-1091.

[28] Dolan RD, McSorley ST, Horgan PG, et al. The role of the systemic inflammatory response in predicting outcomes in patients with advanced inoperable cancer: Systematic review and meta-analysis. Crit Rev Oncol Hematol. 2017; 116:134-146.

[29] Templeton AJ, McNamara MG, Seruga B, et al. Prognostic role of neutrophil-to-lymphocyte ratio in solid tumors: A systematic review and meta-analysis. J Natl Cancer Inst. 2014;106: u124.

[30] Mancuso ME, Santagostino E. Platelets: Much more than bricks in a breached wall. Br J Haematol. 2017; 178:209-219.

[31] Thomas MR, Storey RF. The role of platelets in inflammation. Thromb Haemost. 2015; 114:449-458.

[32] McFadyen JD, Kaplan ZS. Platelets are not just for clots. TRANSFUS MED REV. 2015; 29:110-119.

[33] Ceelen W. HIPEC with oxaliplatin for colorectal peritoneal metastasis: The end of the road?. Eur J Surg Oncol. 2019; 45:400-402.

[34] Klaver $\mathrm{YL}$, de Hingh $\mathrm{IH}$, Boot $\mathrm{H}$, et al. Results of cytoreductive surgery and hyperthermic intraperitoneal chemotherapy after early failure of adjuvant systemic chemotherapy. J SURG ONCOL. 2011; 103:431-434.

[35] Esquivel J, Lowy AM, Markman M, et al. The american society of peritoneal surface malignancies (ASPSM) multiinstitution evaluation of the peritoneal surface disease severity score (PSDSS) in 1,013 patients with colorectal cancer with peritoneal carcinomatosis. ANN SURG ONCOL. 2014; 21:4195-4201.

[36] Demey K, Wolthuis A, de Buck VOA, et al. External validation of the prognostic nomogram (COMPASS) for patients with peritoneal carcinomatosis of colorectal cancer. ANN SURG ONCOL. 2017; 24:3604-3608. 